\title{
ALEXANDER VON HUMBOLDT ET LA CONSTRUCTION FRANCO-ALLEMANDE DE L'AMÉRIQUE LATINE
}

ESPAGNE, Michel ${ }^{1}$

RÉSUMÉ : Ecrivain allemand francophone travaillant sur l'Amérique latine dont il a contribué à fonder l'identité politique et scientifique Alexander von Humboldt qui a travaillé 20 ans dans le milieu des savants parisiens à la préparation éditoriale de son Voyage aux régions équinoxiales du nouveau continent (30 volumes en français) incarne un transfert culturel triangulaire paradigmatique. Le présent article vise à analyser les mécanismes de naturalisation en France de Humboldt à travers deux revues qui accompagnent la diffusion de son œuvre les Annales et Nouvelles Annales des Voyages et la Revue des deux mondes, cette dernière plus particulièrement centrée sur l'acclimatation de la grande œuvre en langue allemande le Cosmos. On a pu de même reconnaître jusque dans des œuvres fondamentales du modernisme brésilien des réminiscences du texte humboldtien sur la nature d'Amérique du Sud.

MOTS-CLÉS : Alexander von Humboldt ; Annales des voyages ; transferts culturels triangulaires ; histoire du livre ; constructions identitaires.

\section{ALEXANDER VON HUMBOLDT AND THE FRENCH-GERMAN CONSTRUCTION OF LATIN AMERICA}

\begin{abstract}
As a German author writing mostly in French Alexander von Humboldt afforded a contribution to Latin American political aesthetical and scientific identity. 20 years long he worked in Paris in the circle of the famous French scholars of his time for the edition of his Personal Narrative of Travels to the Equinoctial Regions of the New Continent during the years 1799-1804 (30 volumes in French) and embodied therefore a paradigmatic cultural transfer between three cultural areas. The
\end{abstract}

\footnotetext{
${ }^{1}$ Spécialiste de l'histoire des échanges culturels au XVIII ${ }^{\mathrm{e}}$ siècle et XIX ${ }^{\mathrm{e}}$ siècle, chercheur et directeur du labex TransferS (ENS-Collège de France-CNRS).
} 


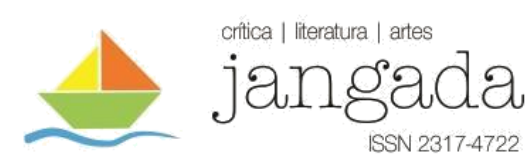

present paper aims to observe the acclimatization of the German Humboldt in France on the basis of two journals Annales et Nouvelles Annales des Voyages et Revue des deux Mondes. The last one focuses more on the main book published in German Cosmos. Even in major works connected with Brazilian modernism reminders of Humboldt's description of Latin American landscape remain perceptible.

KEYWORDS: Alexander von Humboldt; Annales des voyages; triangular cultural transfers; history of the book; identity constructions.

Par transfert culturel (ESPAGNE, 2012) on entend la dynamique de construction d'un système culturel à partir d'importations auxquelles sont ajoutées de nouvelles dimensions sémantiques qui transforment les objets importés. Le résultat obtenu ayant la même valeur que l'original la notion d'influence perd de sa signification et la dynamique du transfert s'oppose à la mise en regard plus statique des comparaisons. Le rôle des médiateurs est fondamental même s'ils représentent une frange réduite de la population des aires culturelles mises en contact. Si l'on s'intéresse à la catégorie des médiateurs la figure d'Alexander von Humboldt, dont le romaniste Ottmar Ette a fait le héros d'une circulation permanente entre les cultures (ETTE, 2019), mérite une attention particulière. Il s'agit en effet d'un savant allemand de langue française apportant des composantes identitaires à l'Amérique latine tout en l'insérant dans un monde globalisé et faisant communiquer entre elles les diverses sciences qui permettent la description d'un continent $^{2}$. Il serait trop simple de voir en Alexander von Humboldt un écrivain allemand qui aurait été reçu en France avec une bienveillance particulière ou avec un sentiment de parenté secrète. Il était plutôt un auteur français d'origine prussienne ou un scientifique allemand de langue française. Il appartenait à cette catégorie négligée d'auteurs ou d'érudits difficiles à classer dans un contexte national. Le fait qu'il représente un discours scientifique européen ne doit pas seulement être affirmé, mais peut aussi être prouvé dans le détail. Sa participation à des revues importantes et l'image de lui-même qu'il n'a cessé de cultiver dans ces revues montrent comment la science transnationale qu'il appelait de ses vœux a pu se constituer. Si l'on veut rendre justice à la perspective de Humboldt, il faut passer du problème de la réception à celui d'un discours transnational et de son émergence. Les Annales ou Nouvelles Annales des

\footnotetext{
${ }^{2}$ Parmi nos publications antérieures sur la question voir Michel Espagne, Momente der deutsch-französischen Weltvermessung um Alexander von Humboldt. In: David Blankenstein, Ulrike Leitner, Ulrich Päßler und Bénédicte Savoy, «Mein zweites Vaterland». Alexander von Humboldt und Frankreich, De Gruyter, Berlin, 2015, p. 71-82. Voir aussi Id. : La sociabilité européenne des frères Humboldt. Paris : Éditions Rue d’Ulm, 2016.
}

Jangada| ano 9, nr. 17, jan/jun, 2021 | ISSN 2317-4722 194 | P á g in a 
voyages, de la géographie et de l'histoire, publiées à partir de 1809, qu'il a co-éditées pendant un certain temps, et la Revue des deux mondes, publiée à partir de 1829, peuvent servir de guide.

Dans la première moitié du XIXe siècle, Paris est resté un centre de la vie scientifique en Europe. La situation n'a pas changé jusqu'en 1850, lorsque les universités allemandes ont récolté les conséquences positives de la réforme humboldtienne. L'avance scientifique du premier $\mathrm{XIX}^{\mathrm{e}}$ siècle est due aux nombreuses institutions scientifiques parisiennes, de l'Institut de France au Jardin des plantes. Elles sont alors encore à leur apogée. Il n'est donc pas rare que des universitaires allemands complètent leur formation sur les bords de la Seine. Alexander von Humboldt, qui a travaillé 20 ans en France à la préparation éditoriale de son livre Voyage aux régions équinoxiales du nouveau continent (30 volumes en français) qui constitue l'essentiel de son œuvre et représente le mieux la curiosité scientifique qui attire de nombreux jeunes Allemands à Paris, où ils profitent de la mise en réseau des scientifiques et d'un milieu global très stimulant pour l'élargir dans la mesure du possible. Une description détaillée des scientifiques allemands établis à Paris dans le premier tiers du XIXe siècle a déjà été élaborée, en tenant compte de la durée du séjour de chaque personne, de la pondération des sujets individuels et des publications (KANZ, 1997). On assiste à la construction d'une couche allemande dans la formation de la mémoire culturelle française. Certains Allemands travaillent également comme traducteurs et traduisent des travaux importants de chercheurs germanophones vers le français. Un exemple est fourni par Heinrich August Vogel, qui a travaillé à Paris de 1802 à 1815 et a fait campagne pour la publication d'ouvrages chimiques en traduction française. Georges Cuvier, originaire de l'enclave allemande en France Mömpelgard/Monbéliard et diplômé de la Karlsschule de Stuttgart, fut dès le départ un interlocuteur classique des invités allemands. Alexander von Humboldt l'a complété ou remplacé dans cette fonction. Par exemple, il a présenté le jeune chimiste Justus von Liebig à son ami Joseph-Louis Gay-Lussac et lui a donné l'opportunité de s'installer à Paris pendant plusieurs mois. Le zoologiste Christian Gottfried Ehrenberg est entré en contact avec les scientifiques français grâce à la médiation active de Humboldt. Celui-ci a permis à l'égyptologue allemand Richard Lepsius de séjourner à Paris. Il l'a même aidé à obtenir un poste de professeur après son voyage en Égypte et l'a promu en aidant à la diffusion de ses recherches. Cependant, Humboldt semble avoir été beaucoup plus hésitant que Cuvier, par exemple, dans ses tentatives de médiation entre deux paysages scientifiques. Contrairement à de nombreux Allemands, il traduisait à peine. Les contacts sociaux en dehors de l'académie étaient bien sûr tout aussi importants pour élargir le réseau personnel des jeunes savants et Alexander von 
Humboldt n'a pas hésité à recourir au pur divertissement de salon lors de la conquête sociale du monde parisien. La maitrise de la langue était un facteur favorable et il ne faut pas oublier que Marie Elisabeth Colomb, la mère de Humboldt, d'une famille de Huguenots, lui a enseigné le français comme deuxième langue maternelle. Humboldt, qui était membre correspondant depuis 1804 et membre associé de l'académie depuis 1810, malgré le contexte peu favorable du premier Empire devait être rétrospectivement perçu comme un Allemand, mais à son époque il se voyait davantage comme un scientifique européen.

Comme le soulignait Ulrich Päßler il y a quelques années dans son livre Un diplomate des forêts de l'Orénoque (2009), la présence scientifique de Humboldt à Paris était également une question diplomatique. Le fait que la description de l'Égypte menée par Edme François Jomard, à la recherche duquel Humboldt aurait aimé participer, ait servi de modèle au récit du voyage en Amérique, est à voir comme un ancrage politique. L'exploration de l'Égypte, entreprise par un collectif d'érudits, semble avoir suscité une description parallèle, quoique unique, de l'Amérique par le seul Humboldt qui élargit un transfert franco-allemand à tout un continent. À ce niveau-là aussi la description de l'Amérique méridionale, inspirée dans son principe par la description de l'Egypte, correspond à un transfert culturel, pratiqué par le francoallemand Humboldt.

Honoré en Amérique latine en raison notamment de son monumental Voyage aux régions équinoxiales du nouveau continent rédigé dans la langue alors la plus répandue dans le monde savant de l'époque et qui donnait au continent une identité à la fois historique anthropologique et géographique voire esthétique, Humboldt pouvait apparaître aussi comme l'inspirateur d'un des principaux révolutionnaires d'Amérique latine, Simon Bolivar, qu'il avait rencontré à plusieurs occasions en Europe, à Paris et à Rome ${ }^{3}$. Ses publications assuraient la reconnaissance européenne de l'Amérique et donnaient des instruments aux hommes politiques américains eux-mêmes ; ses liens avec Bolivar donnaient un écho américain à ses conceptions des indépendances. Il conviendrait de nuancer la reconnaissance des latino-américains selon les régions mais elle est à la fois de nature politique et identitaire. Le monument à Humboldt qui orne le Parque de Mayo à Quito en Equateur est certainement lié à la description de la nature de l'Equateur dans la Vue des cordillères mais par là Humboldt fait partie des artisans d'une auto-représentation de l'espace national et donc aussi de l'identité politique du pays (DURISCH-

\footnotetext{
${ }^{3}$ Sur Bolivar et Humboldt voir notamment : MINGUET, Charles. Alexandre de Humboldt - Historien et géographe de l'Amérique espagnole (1799-1804). Paris, Maspero 1969 ; SAURAT, Gilette. Simon Bolivar le libertador. Paris : Grasset, 1990 ; VAYSSIÈRE, Pierre. Simon Bolivar- Le rêve américain, Paris, Payot 2008.
} 


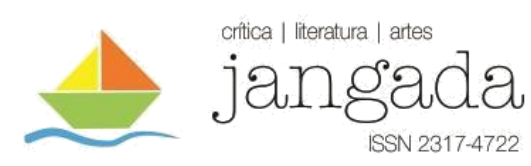

ACOSTA, 2009). Il n'en va pas différemment au Venezuela où divers toponymes, en particulier une montagne rappellent le voyageur (RODRIGUEZ, 2009). Et au Mexique l'Essai sur la Nouvelle Espagne a été perçu comme une célébration des richesses d'un pays, comme si le voyageur allemand faisait découvrir aux Mexicains, statistiques à l'appui, quel bien-être pourrait être le leur après un tournant politique. Carlos Pereyra l'historien mexicain pouvait même voir en Humboldt un fondateur allemand des sciences sociales sur le continent américain (COVVARUBIAS, 2009).

L'attitude de Humboldt à l'égard de la vie intellectuelle internationale au sens large est également significative. À Paris il a été en contact avec l'architecte allemand du Cirque d'Hiver ou de la Gare du Nord, Jakob Ignaz Hittorf, avec qui il a visité l'atelier du peintre Gérard, et il a connu les archéologues Raoul Rochette et Jean-Antoine Letronne. Dans la controverse sur la polychromie, il s'est rangé du côté de Hittorf. Quand l'helléniste berlinois August Böckh a eu besoin de manuscrits parisiens, Humboldt lui a obtenu les copies nécessaires. Sans se limiter aux géographes, il s'intéresse aussi aux sciences humaines en général. Par exemple, il s'est occupé de la réception des publications de son frère Wilhelm en France. Bien au-delà de la science, Humboldt entretient des relations avec les principales figures de la vie politique française sous la monarchie de juillet, comme Thiers et Guizot. Il aurait soutenu Victor Cousin dans son rapport sur l'état du système éducatif allemand. Humboldt est autant une figure politique qu'une figure scientifique de l'histoire culturelle franco-allemande dans la première moitié du siècle. Il rédige des rapports diplomatiques et tente surtout de faire connaître les résultats de la recherche prussienne en France. Ses bonnes relations avec des hommes politiques importants lui ont permis de défendre à l'occasion un scientifique qu'il appréciait, même après son retour à Berlin. Par exemple, il s'est employé à soutenir Jean Baptiste Boussingault (18021887), qui a passé une décennie en Amérique du Sud avant de se lancer dans une carrière de chimiste en France. La coopération transnationale envisagée par Humboldt était bien entendu une contribution scientifique au rapprochement politique mutuel des pays européens. Humboldt s'est par conséquent efforcé de renforcer cette collaboration dans le domaine du géomagnétisme, qui était un préalable disciplinaire à la description de la terre.

Humboldt, qui, nous l'avons dit, maîtrisait le français comme sa langue maternelle et considérait sa description du paysage américain comme un défi stylistique et une œuvre d'art, peut difficilement être analysé du point de vue trop unilatéral de la réception d'un écrivain de langue étrangère. Il doit néanmoins être aussi perçu comme un homme de lettres français. On pourrait peut-être partir d'une position intermédiaire, d'une fonction de pont qui ne peut être 


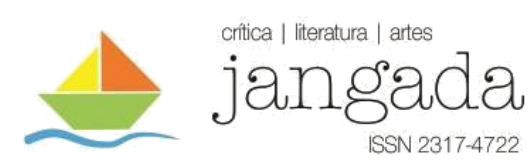

assignée à aucune des deux rives, mais qui a beaucoup facilité la communication entre les deux rives. Le nécrologue plaide toutefois pour une appartenance française: «C'est à Paris, pendant un séjour de plus de vingt ans qu'il fit dans notre capitale, que M. von Humboldt publia cette magnifique série de travaux. Dans l'intervalle, il trouvait le temps de faire des expériences sur l'anatomie, la physiologie et la chimie. Partageant ses journées entre le cabinet de Cuvier, et le laboratoire de Gay-Lussac, en contact permanent avec nos plus illustres savants, Laplace, Biot, Brongniart, Thénard, etc., il considérait Paris comme sa véritable patrie scientifique ${ }^{4}$. »

Ce n'est pas la réception de Humboldt mais les mécanismes de naturalisation, la disparition des frontières scientifique qui doivent être examinés ici, deux revues les Annales et Nouvelles Annales des Voyages fournissant un guide disciplinaire et la Revue des deux mondes, si centrale pour le XIXe siècle, un guide plus général.

La revue Annales des voyages, de la géographie et de l'histoire a été fondée en 1807. Le premier éditeur fut le géographe d'origine danoise Conrad Malte-Brun (1775-1826). Immigré à 24 ans en France il a publié une description en six volumes de toutes les régions du monde en 1810 et a fondé la Société géographique française en 1821. Il a été le premier secrétaire général de cette société. Humboldt a contribué aux premiers numéros. Ce sont des fragments de ses œuvres ultérieures. En 1809 a été publié un essai sur la canne à sucre à Tahiti. Un extrait des Vues de la nature sur les tribus des environs de Caracas supposées manger la terre est également imprimé en 1809. La même année paraît un extrait du Voyage à l'intérieur de l'Amérique. Il donne à Humboldt l'occasion de familiariser le public parisien avec l'ensemble de son ambitieux projet. La présentation au public parisien de l'espace américain s'opère pas à pas. Le travail a été annoncé dans une série d'extraits et d'articles. Il s'agissait d'abord de la répartition géographique des espèces végétales. En 1810 fut évoquée la manière dont Humboldt envisageait l'aspect pictural de la Cordillère américaine et l'art des Indiens. Il s'agissait évidemment de rendre l'ensemble du livre de voyage séduisant aux yeux des futurs lecteurs avant que l'entreprise ne soit très avancée. La journal a essayé de souligner d'emblée le large spectre du récit de voyage en publiant un long extrait de l'essai politique sur la vice-royauté de la Nouvelle-Espagne en 1810. Cette fois, il s'agissait d'aspects politico-historiques.

À partir de 1819, les Nouvelles Annales paraissent sous la direction principale de MalteBrun et de Jean-Baptiste Eyriès (1767-1845) qui appartenait également à la Société de géographie, parlait, dit-on, 9 langues et avait traduit de nombreux récits de voyage en français.

\footnotetext{
4 Nouvelles annales 1859, T. 162., p. 215.
}

Jangada | ano 9, nr. 17, jan/jun, 2021 | ISSN 2317-4722 


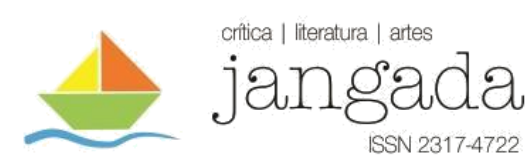

La renommée européenne de Humboldt doit beaucoup aux deux hommes et à leur revue. Il faut également mentionner ici l'éditeur Frédéric Schoell (1756-1833), qui avait travaillé comme fonctionnaire prussien avant de s'installer à Paris comme libraire et éditeur. Il était l'un de ces éditeurs de langue allemande qui ont joué un rôle majeur dans la publication de la littérature scientifique à Paris au XIXe siècle. La principale préoccupation des rédacteurs des Nouvelles Annales était de faire connaître le Voyage aux régions équinoxiales du nouveau continent. La plupart du temps, les extraits de passages particuliers du voyage ont été rassemblés par les principaux rédacteurs de la revue. En 1819 parut par exemple, le rapport sur le tremblement de terre de Caracas. Dans son texte d'accompagnement, Malte-Brun a souligné les aspects aventureux du voyage afin de rendre la lecture plus passionnante: « M.de Humboldt s'enfonce enfin dans les contrées incultes et sauvages qu'arrose l'Orénoque; c'est ici que son voyage prend le caractère romanesque et aventureux tant goûté par la plupart des lecteurs ; plus de sites superbes, plus de luxe, plus de maîtres et d'esclaves; les singes, les tigres, les Indiens et les missionnaires, telle est la société de notre voyageur, soit qu'il descende dans une frêle-barque le large Orénoque ou qu'il erre à pied d'une cabane à l'autre ${ }^{5}$. » Parfois, les extraits sont particulièrement volumineux. En 1826, plus de 50 pages de la première partie de la description de l'île de Cuba ont été imprimées. En 1826, le journal mentionne l'accueil enthousiaste du Voyage et imprime des extraits de la Géographie des plantes publiée par Humboldt et Karl Sigismund Kunth. En 1827 vint le rapport sur la province d'Antioquia et la découverte de gisements de platine dans cette province. Cette description a servi de prétexte à la publication d'une lettre envoyée d'Amérique par Boussingault. La même année, fut publiée une présentation détaillée de la République du Guatemala. Ces éléments du voyage en voie de rédaction sont si importants pour la politique d'édition des maisons d'édition concernées que Humboldt a fait insérer une lettre ouverte à l'éditeur Gide lorsque le voyage prévu en Russie menaça de retarder la publication du Voyage aux régions équinoxiales du Nouveau continent ${ }^{6}$. Les Vues de la nature sont également un thème majeur dans la production littéraire de Humboldt. Lorsque l'éditeur des Nouvelles Annales, Eyriès, traduisit le livre en 1828, un compte rendu fut publié par Philippe François Lasnon de la Renaudière, qui à l'époque codirigeait les Nouvelles Annales elles-mêmes ${ }^{7}$. La production scientifique de Humboldt était évidemment si importante pour la revue qu'elle se mettait sans limites au service de sa diffusion. Chaque lecteur de la revue était

\footnotetext{
${ }^{5}$ Nouvelles Annales, 1819, T.3, p. 213.

${ }^{6}$ Nouvelles Annales, 1829/01, T. 41, p. 380.

${ }^{7}$ Nouvelles Annales, 1828/01, T. 37, p. 353.
}

Jangada| ano 9, nr. 17, jan/jun, 2021 | ISSN 2317-4722 


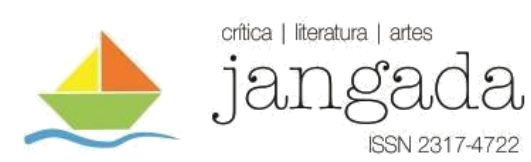

aussi bien informé que le lecteur du Voyage ou de l'un des autres livres de Humboldt. Après la première recension des Vues de la nature, une seconde parut en 1856, cette fois relative à la $3 \mathrm{e}$ édition et signée par le diplomate et écrivain Adolphe de Circourt $^{8}$. Cette fois, le critique a souligné la qualité littéraire du livre, répondant à une tendance de plus en plus marquée dans la réception des livres de Humboldt. Circourt et La Renaudière, Eyriès et Malte-Brun n'appartenaient pas au réseau des amis français les plus célèbres de Humboldt. Outre les relations avec Arago et Gay-Lussac, il ne faut pas sous-estimer l'importance de ces figures littéraires, dévouées sans réserve à la réputation de Humboldt. Ils ont été les premiers lecteurs de ses écrits et les véritables vecteurs de sa renommée. Ce qui est remarquable dans la sélection d'extraits publiés, c'est, d'une part, l'utilisation systématique de tableaux et de figures qui garantissent le sérieux de l'argumentation et confortent Humboldt dans son activité d'arpenteur du monde, et d'autre part, la variété des sujets de recherche considérés comme pertinents. L'histoire politique de l'île de Cuba n'est pas moins pertinente que le développement de l'air froid lors de l'ascension du Chimborazo. Bien que Humboldt ait professé à plusieurs reprises l'empirisme, sa tendance au holisme est restée indéniable. C'est précisément cette capacité à combiner des observations quantifiables avec des analyses en sciences humaines qui a impressionné Franz Boas, le fondateur de l'anthropologie américaine dans la géographie de Humboldt, conçue comme premier niveau d'ethnologie. Le fait que la diversité des phénomènes naturels, qu'ils soient d'origine humaine ou physique, devait être ramenée à un dénominateur commun, a aussi quelque chose à voir avec la philosophie naturelle.

Lorsque Humboldt entreprit son voyage en Sibérie en 1829 9ESPAGNE, 2011, p. 107122). et voulut confronter les hypothèses et observations formulées en Amérique du Sud à l'exploration de l'Asie centrale, les Nouvelles Annales suivirent la nouvelle exploration avec la même attention que les récits sur l'Amérique du Sud. Après son voyage relativement court à travers la Sibérie, Humboldt, désormais célébré comme savant, a été autorisé à donner une conférence publique à l'Académie des sciences de Saint-Pétersbourg. Il y énumère les réalisations des géographes russes et met particulièrement au crédit de la famille Parrot, d'origine française mais établie à l'université germanophone de Dorpat d'avoir réussi une ascension du mont Ararat. Les scientifiques russes et les Allemands de Russie parmi eux ont été félicités. Humboldt a également profité de cette occasion pour demander aux collègues russes, qui disposaient d'un vaste champ d'expérience, d'étudier de plus près le magnétisme et

${ }^{8}$ Nouvelles Annales, 1856, T 149, p. 340-366.

Jangada | ano 9, nr. 17, jan/jun, 2021 | ISSN 2317-4722

200 | P á g i n a 


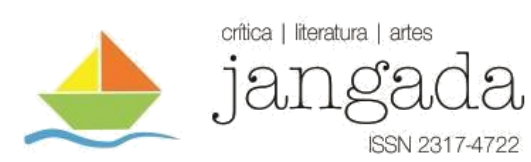

le niveau de l'eau dans la mer Caspienne. Le texte intégral de la conférence pouvait être lu dans la première édition des Nouvelles Annales pour l'année 1830. À partir de 1830 on trouve des contributions sur l'Asie centrale. En 1830, par exemple, fut publié un long traité de Humboldt sur les éruptions volcaniques et les chaînes de montagnes en Asie centrale ${ }^{9}$. Construisant dans l'espace franco-allemand une identité scientifique, politique et esthétique de l'Amérique latine, Humboldt avait besoin d'une incursion en Asie centrale pour que par complémentarité la découverte de l'Amérique latine ait la qualité d'une expérience globale du monde.

La construction d'un mythe de Humboldt commence donc dans les essais des Annales et Nouvelles annales des voyages. Même avant la mort de Humboldt, la revue rappellera avec émotion qu'une ville du Kansas et une autre ville de Californie ont été baptisées du nom de Humboldt. Le géographe a également donné son nom à une rivière et à un glacier du Groenland. Il ne s'agissait pas seulement de mentionner les toponymes qui existaient. La référence à ces noms fut cependant un moment dans la construction du mythe qui se déroula dans le contexte parisien des auteurs des Annales.

Alors que les Annales ont assuré la renommée de Humboldt en publiant des extraits de son Voyage ou ses Vues de la nature, et plus tard même en annonçant les résultats de recherches en Asie centrale, la Revue des deux mondes a poursuivi cette diffusion en faisant connaître le Cosmos à un large public. Le lectorat des Annales était composé de professionnels. La Revue des deux mondes, quant à elle, s'adressait à tous les Français éduqués et bénéficiait d'une réception optimale. Elle se situait au cœur de la vie littéraire et intellectuelle de la Monarchie de juillet et traitait de tous les domaines possibles de la connaissance, de la littérature aux sciences humaines et sociales qui se dessinaient progressivement. Les revues techniques étant encore très rares, elle assurait la diffusion de nouvelles connaissances dans tous les domaines du savoir n'appartenant pas directement aux sciences physiques. Le Voyage a été écrit et publié directement en français, Humboldt utilisant tous les moyens disponibles pour affirmer ses ambitions stylistiques. Le public avait découvert une œuvre à mi-chemin entre littérature et sciences, et ce double visage avait visiblement facilité la réception de l'ouvrage. Le Cosmos, qui représentait en quelque sorte un résumé de toutes les recherches de Humboldt, a eu une histoire différente.

Le gros livre devait être traduit. L'entreprise s'est déroulée s'est déroulé dans un milieu d'amis et connaissances. Le principal traducteur était un élève d'Arago, Hervé de Faye (1814-

\footnotetext{
${ }^{9}$ Nouvelles Annales ,1830/ 10, T. 48, p. 217.

Jangada | ano 9, nr. 17, jan/jun, 2021 | ISSN 2317-4722 
1902), qui a lui-même publié un manuel d'astronomie et un traité sur la représentation de la création du monde, la cosmogonie. Avec Hervé de Faye disparaît le mur entre le transfert et la tentative de s'affirmer comme penseur original de l'œuvre traduite. Arago et l'archéologue Joseph Daniel Guigniaut avaient eux-mêmes traduit certains passages ou, dans le cas d'Arago, conseillé le traducteur principal Faye. L'éditeur était dès le départ la «librairie de Gide» qui, dans le catalogue de 1841, annonçait déjà un nombre considérable de publications de Humboldt, disponibles ou sous presse. La librairie de Gide aimait publier de la littérature de voyage et éditait, entre autres, les Nouvelles Annales. Humboldt y publia une introduction au Cosmos en 1845. Il en a profité pour traduire lui-même ou plutôt, comme le dit l'éditeur du journal, il a repensé en français ce qu'il avait conçu en allemand. Cette introduction correspondait à une présentation de sa propre conception de la nature et de la philosophie naturelle : « La nature, considérée rationnellement, c'est-à-dire soumise dans son ensemble au travail de la pensée, est l'unité dans la diversité des phénomènes, l'harmonie entre les choses créées dissemblables par leur forme, par leur constitution propre, par les forces qui les animent, sont différentes; c'est le tout (to pân) pénétré d'un souffle de vie ${ }^{10}$. » L'examen intellectuel de la nature n'a cependant lieu que dans l'hémisphère nord, comme si certains continents étaient plus favorables au progrès de la raison que d'autres. Les germes de la civilisation sous les tropiques ont d'abord dû être importés, pense Humboldt, et la colonisation aurait eu un impact majeur sur ce développement. Humboldt semble vouloir trouver des aspects positifs à la colonisation. Cette histoire aussi appartenait au système englobant de la nature. Il était important pour lui que la variété des phénomènes puisse être réduite à des archétypes. Les variations produites sur cette base lui rappellent la théorie goethéenne des métamorphoses. Afin de pouvoir reconnaître les lignes directrices des métamorphoses, on ne pouvait pas se plonger dans la représentation de tous les détails. Après la construction du bâtiment, les échafaudages pouvaient être enlevés. Les Allemands seraient trop obsédés par les questions de détail. Et Humboldt prend ouvertement parti pour une rhétorique française dans la présentation des résultats de sa recherche. La France et l'Allemagne peuvent cependant se vanter de ce qu'avec la géographie de Carl Ritter et la représentation du système du monde par Laplace, elles ont apporté des contributions décisives à l'exploration du cosmos qu'il avait toujours à l'esprit. Surtout, Humboldt se défendit contre le soupçon d'avoir écrit un ouvrage philosophico-spéculatif. D'un autre côté, considérer l'univers comme un système était important pour lui. Il se réclama donc d'un « empirisme raisonné ».

${ }^{10}$ RDDM, 1845/tome 12, 14e année, p. 752. 


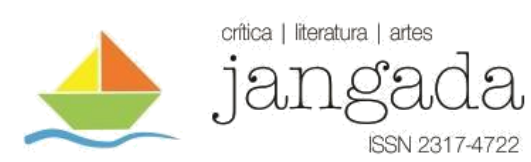

Les phénomènes individuels peuvent être perçus comme isolés, mais ils peuvent encore cacher le germe d'une grande découverte et doivent de toute façon être mis en harmonie avec le système mondial, avec l'ensemble.

Après cette introduction, signée par Humboldt lui-même, l'objectif principal de la Revue des deux mondes était de familiariser le public avec les aspects clés de l'œuvre elle-même. Des parties plus longues du Cosmos ont été résumées ou citées en détail et commentées en même temps. Jean Louis Armand Quatrefages de Bréau, qui avait enseigné comme zoologiste à l'université de Toulouse avant d'être nommé à une chaire d'ethnologie au musée et de publier un livre sur l'unité de l'humanité en 1861, écrivit en 1846 son premier long article sur la description physique du monde dans le Cosmos. Quatrefages a commencé avec un résumé de la biographie de Humboldt et a raconté ses tentatives de se joindre à l'exploration prévue de l'Égypte. Dans les sciences particulières, Humboldt ne pouvait pas rivaliser avec des gens comme Lavoisier, Jussieu, Cuvier, mais en tant que "physicien du globe", en tant qu'auteur d'une description physique du monde, personne ne pouvait atteindre à son niveau. Quatrefages met à son crédit la dimension poétique de sa perception du monde: Humboldt voit non seulement avec l'esprit mais avec les yeux corporels.

Quelques années plus tard, le mathématicien, physicien et astronome Jacques Babinet, qui avait travaillé avec Ampère sur l'électricité et le magnétisme, consacra un long article à la description humboldtienne du ciel. Ce qui était important pour Babinet, c'était le fait que Humboldt avait suggéré aux scientifiques qu'il était nécessaire de voyager. On pourrait lui reprocher de s'être trop engagé au début du Cosmos dans la métaphysique allemande, qui mélangeait observation et métaphysique. Mais il avait mis en relation l'univers et l'âme humaine : Babinet admirait le tableau des corps célestes conçu par Humboldt. Il se considérait comme un connaisseur des étoiles filantes et pouvait assurer au lecteur que personne n'avait fourni une enquête plus détaillée que Humboldt dans les chapitres correspondants de son Cosmos

Contrairement aux Nouvelles Annales, les essais de Humboldt dans la Revue des deux mondes ne sont pas une compilation d'extraits. Les contributeurs s'autorisaient de temps à autre des tons critiques, mais prenaient surtout position sur un bilan scientifique dont ils se sentaient très familiers du fait de leur propre formation et qu'ils ne considéraient nullement comme une importation de l'étranger. Avec Quatrefages et Babinet ou Auguste Laugel, s'élargit le réseau des connaissances françaises de Humboldt qui, comme les traducteurs Faye et Eyries ou l'éditeur Malte-Brun, ont contribué à la diffusion de son œuvre et au processus de sa pleine naturalisation. 
Alexander von Humboldt devient progressivement une figure de proue de la science européenne, un monument commun à la France, à l'Allemagne et bien sûr à l'Amérique latine. Cette évolution se fait sentir au plus tard à l'annonce de sa mort. La cérémonie funéraire berlinoise a été décrite en détail dans les Nouvelles Annales, comme s'il était important que le public français soit là aussi. La nécrologie faisait plus de 20 pages. Humboldt a été présenté comme l'un des représentants les plus renommés de la science moderne et l'un des plus merveilleux porte-parole de l'esprit humain. Son importance pour la France y est soulignée à plusieurs reprises, par exemple lorsqu'est évoqué le Cosmos: "Grâce aux soins actifs et persévérants de l'éditeur, M. Gide, à qui la science doit la merveilleuse publication des œuvres d'Arago, la littérature française est aujourd'hui en possession, avec le Cosmos, d'un ouvrage vraiment impérissable et qui sera toujours lu avec profit par les gens du monde et par les savants ${ }^{11}$. » Pour exprimer leur chagrin après la mort $\mathrm{d}^{\prime}$ « Aristote moderne », la plupart des maisons de l'Oranienburger Strasse auraient été tendues de drapeaux. Les 600 étudiants de l'université de Berlin ont accompagné le cercueil. La famille du roi de Prusse a entendu le discours funèbre du prédicateur de la cathédrale Hoffmann avant que le défunt ne soit enterré à Tegel à côté de son frère Wilhelm. Il a été constaté que Humboldt n'avait pas rédigé de testament, mais avait plutôt déterminé dans une donation privée qu'il léguerait toute sa fortune à son valet Johann Seifert. Les récompenses dont il avait été honoré toute sa vie gisaient dans un placard, désorganisées. Le ministre d'État Achille Fould a répété dans une adresse à Napoléon III que Humboldt était presque devenu un concitoyen et en tant que tel méritait un honneur spécial. Il érigea une statue dans la galerie de Versailles pour que Humboldt ne soit jamais trop éloigné de ses admirateurs et amis.

Dans un article publié en 1824, Humboldt a tenté de comprendre en analysant la qualité de l'eau et en mesurant la chaleur dans diverses vallées andines pourquoi des goitres apparaissaient souvent la population locale ${ }^{12}$. Bien qu'il n'ait pas tiré de conclusion, il a privilégié l'humidité de l'air comme facteur possible. Il était moins préoccupé par une explication que par une description des faits. Cette perspective ouverte était probablement la raison pour laquelle ses domaines d'intérêt étaient si divers et, de notre point de vue actuel, hétérogènes. En 1838, Humboldt a fourni un rapport détaillé sur deux tentatives pour atteindre

\footnotetext{
${ }^{11}$ Nouvelles Annales, 1859, T. 162, p. 218.

${ }^{12}$ Nouvelles Annales 1824, T 23, p. 338-439.
} 
le sommet du Chimborazo ${ }^{13}$. Le rapport d'environ 40 pages se lit comme la combinaison d'un rapport de recherche sur la pression atmosphérique en altitude ou sur les types de roches et l'histoire de la conquête d'un nouveau sommet par les alpinistes. La constitution géognostique du Chimborazo n'a en rien empêché le bilan esthétique qui a accompagné l'exploration de tous les phénomènes naturels lors du voyage américain: «Telles sont les remarques passagères sur deux ascension du Chimborazo, que je me permets de communiquer aux géologues, simplement telles que je les ai extraites d'un journal de voyage encore inédit. Quand la nature est si majestueuse et si puissante, et que nos efforts sont uniquement consacrés à la science, le discours dans lequel nous les présentons, peut bien se passer de tout ornement ${ }^{14}$. »C'est précisément ce prétendu abandon des moyens rhétoriques qui est un signe de la littérarisation humboldtienne des découvertes scientifiques.

L'ampleur thématique des contributions de Humboldt explique également pourquoi les Nouvelles Annales et la Revue des deux mondes se réfèrent souvent à son autorité pour accroître la force de persuasion des arguments scientifiques. La référence aux œuvres de Humboldt semble ainsi être devenue un topos de la rhétorique scientifique.

Quand, en 1853, une traduction française de l'histoire de Hiouen Thsang (Xuanzang) qui traduisit le canon bouddhiste en chinois - et de ses voyages à travers l'Inde parut dans une traduction fournie par le sinologue Stanislas Julien, la recension des les Nouvelles Annales se référa à la thèse de Humboldt selon laquelle une profonde dépression sépare les montagnes de l'Altaï du Pamir ${ }^{15}$. Bien que Humboldt n'ait rien à voir avec ce voyage, la référence à son interprétation géographique de l'Asie centrale signalait un degré supplémentaire d'érudition. Quiconque se réfère à l'Amérique du Sud ou à l'Asie centrale devait montrer qu'il connaissait Humboldt. Lorsque la Revue des deux mondes publie en 1872 un essai de l'historien Auguste Geffroy (1820-18995) sur l'origine du germanisme, et mentionne un passage de Tacite où la flotte de Germanicus a été exposée à un orage, l'auteur évoque à une déclaration de Humboldt, qui aurait toujours était ravi en lisant le passage. Et lorsque Saint René Taillandier écrivit un essai sur Voltaire en 1865 et voulut donner au lecteur une idée de l'importance que Maupertuis avait au XVIIIe siècle, il compara Maupertuis au charisme de Humboldt dans la seconde moitié du XIX ${ }^{\mathrm{e}}$ siècle.

\footnotetext{
${ }^{13}$ Nouvelles Annales $1838 / 10$, T. 80, p. 5-43.

${ }^{14}$ Ibid, p. 43

${ }^{15}$ Nouvelles Annales 1853, T. 137, p. 265.
} 
On a pu de même reconnaître jusque dans des œuvres fondamentales du modernisme brésilien comme le Macounaïma de Mario de Andrade des réminiscences du texte humboldtien sur la nature amazonienne. L'authenticité primitive de la nature brésilienne serait ainsi en fait l'importation d'une description engagée par Humboldt dans son voyage aux régions équinoxiales. Le perroquet qui garde en mémoire les hauts faits de Macounaïma proviendrait directement du texte du voyageur allemand : «Alors l'homme découvrit dans les frondaisons un perroquet vert au bec doré qui le lorgnait. Il lui dit : donne la patte perroquet !

L'oiseau vint se poser sur la tête de l'homme et ils fraternisèrent. Alors le perroquet se mit à parler dans un parler doux et nouveau, tout nouveau, pareil à un chant et comme le pivori mêlé au miel, il était bon, il avait la saveur captieuse des fruits inconnus de la forêt. «Et dans le silence de l'Ouricoera seul le perroquet préservait de l'oubli la geste et le parler disparus. Seul dans le silence le perroquet conservait les mots et les exploits de notre héros. » (MARIO DE ANDRADE, 1979, p. 246-247) ${ }^{16}$ La référence à des mythes cosmogoniques, à des légendes indiennes rapportant les hauts faits de héros est perceptible dès les premières lignes d'un texte qui se veut l'héritier des cultures autochtones, si éloignées de la littérature lusophone :

« C'est au fin fond de la forêt vierge que naquit Macounaïma, héros de chez nous. Il était noir renoirci et fils de la peur qu'inspire la nuit. Vint un instant où fut si grand le silence pour écouter le clapotis de l'Ouraricoera que l'Indienne Tapianoumas mit bas un bien vilain enfant. Celui-là qu'on appela Macounaïma. » (MÁRIO DE ANDRADE, 1979, p. 25)

En restant au Brésil, on pourrait aussi évoquer l'œuvre centrale que fut le Diadorim (1956) de Guimaraes Rosa), vaste fresque du Sertao qui reprend et transfigure évidemment les légendes et faits de langue locaux mais les fait aussi communiquer avec d'autres espaces littéraires et linguistiques auxquels sa vaste culture permettait à l'auteur d'accéder. De façon plus large l'histoire littéraire du Brésil se constitue en partie en dehors des frontières ou reprend des impulsions venues de l'extérieur (ROZEAUX, 2012) ${ }^{17}$.

Les poètes Joaquim de Sousândrade qui se sert de la Vue des cordillères dans son principal poème (Guesa) et peut-être Pablo Neruda empruntent à Humboldt le cadre de

\footnotetext{
${ }^{16}$ Mario de Andrade, Macounaïma, Paris, Flammarion 1979, p. 246-247. Voir aussi : LIBRANDI ROCHA, Lúcia Ricotta Marília. A Natureza Americana em Humboldt. In : Florema, jan/jun. 2010, p. 7-17.

17 Voir la thèse de Sébastien Rozeaux soutenue en 2012 à l'Université de Lille 3 : La genèse d'un "grand monument national » : littérature et milieu littéraire au Brésil à l'époque impériale (1822-1880).
} 


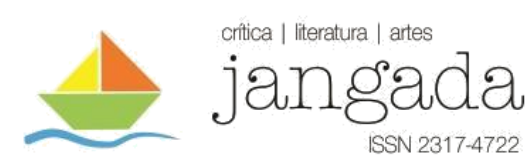

l'imaginaire spatial sud-américain (CLARK ; LUBRICH, 2012). Andres Bello, poète chilien né au Venezuela, dans un poème de 1823 évoque aussi la nature américaine dans les tons de Humboldt qu'il avait directement connu. Quant au poète cubain Jose Maria Heredia, cousin du poète homonyme français il reprend dans son poème En el teocalli de Cholula (1820) les enthousiasmes humboldtiens face aux anciennes cultures méso-américaines.

Humboldt pourrait facilement figurer sur la liste des grands écrivains francophones du $\mathrm{XIX}^{\mathrm{e}}$ siècle, si l'historiographie littéraire réussissait un jour à s'émanciper des frontières nationales. Ses descriptions de la nature sauvage d'Amérique du Sud rivalisent avec l'habileté rhétorique d'un Chateaubriand. Une constante dans la perception du monde par Humboldt est qu'il a voulu relier les phénomènes mondiaux les plus éloignés les uns aux autres. Le vrai gain était le changement constant de perspective. Ce sens du changement systématique de perspective et de la mise en convergence d'objets différents est le plus susceptible d'être observé lorsque l'on pose la question des classifications nationales.

Les contiguïtés entre l'histoire littéraire et l'anthropologie sont chez lui d'autant plus fortes que l'on peut considérer nombre d'anthropologues du point de vue de leur relation à l'écriture. Car le géographe Humboldt est aussi une source pour les anthropologues. On peut retenir par exemple une des figures fondatrices de cette discipline au XIXe siècle, celle de Theodor Waitz, auteur d'une Anthropologie der Naturvölker en six volumes. Waitz, qui n'a guère voyagé et recherche tous les indices d'une unité de l'espèce humaine à travers l'histoire des peuples et de leurs métissages, cite fréquemment Humboldt comme une autorité. La répugnance des peuples à l'état de nature à porter des vêtements est, selon Waitz, attestée par Humboldt (WAITZ, 1859, t. I, p. 358).

S'il est une caractéristique fondamentale de l'œuvre d'Alexander von Humboldt c'est bien d'envisager le monde à la fois comme un système global et comme un système en perpétuel mouvement, au moins pour ce qui est de la géographie humaine. La globalité du monde pour le géographe qu'est Humboldt va de la structure physique du monde, de ses caractéristiques géologiques ou climatiques en passant par la faune et la flore jusqu'à l'organisation des sociétés humaines. Et le tout est abordé non seulement avec l'œil du savant mais avec celui du peintre ou de l'écrivain soucieux de descriptions poétiquement adéquates. L'universalisme de Humboldt est déjà marqué par le fait qu'il s'agit d'un géographe allemand écrivant en français une somme à la fois scientifique et littéraire sur un continent jusque-là méconnu mais dont il va créer, pour ainsi dire de l'extérieur, la conscience identitaire. C'est donc dans le cadre de l'Amérique latine qu'il convient plus particulièrement d'observer la méthode mise en œuvre 


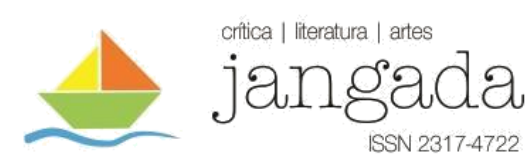

par Humboldt et la manière dont son œuvre peut être analysée comme une interaction entre les cultures.

\section{RÉFÉRENCES BIBLIOGRAPHIQUES}

ANDRADE, Mário. Macounä̈ma. Paris: Flammarion, 1979.

Annales des voyages, de la géographie et de l'histoire

CLARK, Rex; LUBRICH, Oliver. Transatlantic echoes. Alexander von Humboldt in World Literature. Oxford : Berghahn, 2012.

COVVARUBIAS, José Enrique. La recepción de la figura y obra de Humboldt en Mexico 1892-2000. In : HiN, 19-2009.

DURISCH-ACOSTA, Christian. Die Humboldt-Rezeption in Ecuador. Ein kulturgeschichtlicher Beitrag zum ecuadorianischen Nationsbildungsprozess. In : HiN, 192009.

ESPAGNE, Michel. La notion de transfert culturel. In : Revue Sciences/Lettres [En ligne], 1 | 2013, mis en ligne le 01 mai 2012, consulté le 09 juin 2021. URL : http://journals.openedition.org/rs1/219; DOI : https://doi.org/10.4000/rs1.219

. Momente der deutsch-französischen Weltvermessung um Alexander von Humboldt. In: David Blankenstein, Ulrike Leitner, Ulrich Päßler und Bénédicte Savoy, "Mein zweites Vaterland». Alexander von Humboldt und Frankreich, De Gruyter, Berlin, 2015, p. 71-82.

. La sociabilité européenne des frères Humboldt, Paris : Éditions Rue d'Ulm, 2016.

. Le voyage en Russie d'Alexandre de Humboldt. In : Études germaniques, janvier-

mars 2011, p. 107-122.

ETTE, Ottmar Ette. Alexander von Humboldt und die Globalisierung. Das Mobile des Wissens. Berlin : Suhrkamp, 2019.

KANZ, Kai Torsten. Nationalismus und internationale Zusammenarbeit in den Naturwissenschaften. Die deutsch-französischen Wissenschaftsbeziehungen zwischen Revolution und Restauration 1789-1832. Stuttgart: Franz Steiner Verlag, 1997.

LIBRANDI ROCHA, Lúcia Ricotta Marília. A Natureza Americana em Humboldt. In : Florema, jan/jun. 2010, p. 7-17.

MINGUET, Charles. Alexandre de Humboldt - Historien et géographe de l'Amérique espagnole (1799-1804). Paris, Maspero 1969.

PÄßLER, Ulrich. Ein « Diplomat aus den Wäldern des Orinoko ». A. von Humboldt als Mittler zwischen Preußen und Frankreich. Stuttgart: Franz Steiner, 2009.

RODRIGUEZ, José Ángel. El culto a Humboldt en Venezuela. In : HiN, 19-2009.

ROZEAUX, Sébastien. La genèse d'un "grand monument national » : littérature et milieu au Brésil à l'époque impériale (1822-1880). Thèse de doctorat soutenue en 2012 à l'Université de Lille 3.

SAURAT, Gilette. Simon Bolivar le libertador. Paris : Grasset, 1990.

WAITZ, Theodor. Anthropologie der Naturvölker. Leipzig : Friedrich Fleischer, 1859.

VAYSSIÈRE, Pierre. Simon Bolivar- Le rêve américain, Paris, Payot 2008.

Jangada| ano 9, nr. 17, jan/jun, 2021 | ISSN 2317-4722

208 | Pá g in a 


\section{Revues}

Les Nouvelles Annales des voyages, de la géographie et de l'histoire Revue des Deux Mondes 